\title{
Transforming small localized loading into large rotational motion in soft anisotropically-structured materials
}

Rudykh, Stephan, rudykh@mit.edu, Technion - Israel Institute of Technology; Boyce, Mary, Columbia University, United States

\begin{abstract}
Actuation of rotational motion in machines and robotics is generally achieved through highly engineered mechanical or electromechanical devices. As the field of soft robotics develops, there is an emerging and expanding need for novel actuation mechanisms. Here, we show the ability to transform small localized loading into large rotational motion via the design of soft anisotropically structured composite materials. The transformation mechanism governing the rotational actuation capitalizes on the underlying coupling of shear and normal modes of stress and strain in anisotropic materials together with the ability of the soft material to locally undergo large deformation $[1,2]$. The transformation behavior is further shown to be highly tuneable through selection of the microstructure as demonstrated through simulations and through experiments on multimaterial 3D-printed prototypes of soft composite materials with layered microstructures [2]. The study provides guidelines for designing soft anisotropic materials with tailored performance. The mechanisms of large controllable actuation can be used for macro-, micro- and nanoactuators and sensors. The findings can be also used for developing simple techniques for obtaining information on anisotropy, and microstructures of materials at small scales.
\end{abstract}

\section{REFERENCES:}

[1] Rudykh, S., Boyce, M.C. Analysis of Elasmoid fish imbricated layered scale-tissue systems and their bioinspired analogues at finite strains and bending. IMA Journal of Applied Mathematics. 2014 (in press). DOI: 10.1093/imamat/hxu005

[2] Rudykh, S., Boyce, M.C. Transforming small localized loading into large rotational motion in soft anisotropically-structured materials. Advanced Engineering Materials. 2014 (in press). 